\title{
Disseminated Histoplasmosis Post-IL6 Inhibitor Use in A COVID-19 Patient
}

\author{
Premmapassan Krishnamurthy ${ }^{1}$, Brijesh Sharma', Desh Deepak ${ }^{1}$ Shailaja Shukla ${ }^{3}$, Vishakha Arya ${ }^{3}$, \\ Anuradha Chowdhary ${ }^{4}$
}

\author{
${ }^{1}$ Department of Medicine, Dr. Ram Manohar Lohia Hospital, New Delhi, India \\ ${ }^{2}$ Department of Respiratory Medicine, Dr. Ram Manohar Lohia Hospital, New Delhi, India \\ ${ }^{3}$ Department of Pathology, Lady Hardinge Medical College \& Smt. S. K. Hospital, New Delhi, India \\ ${ }^{4}$ Department of Mycology, Vallabhbhai Patel Chest Institute, University of Delhi, New Delhi, India
}

\begin{abstract}
We report a case of a 40-year-old male patient who developed a fever one week after recovering from severe COVID-19 illness that had needed treatment with injectable corticosteroids and Tocilizumab. The patient had had maculopapular lesions on his trunk months before contracting COVID-19, but the skin lesions progressed along with the post-covid fever. Detailed workup and biopsy from skin lesions on the face, trunk, and bone marrow revealed Histoplasmosis. This case highlights the possibility of flaring up of Histoplasmosis in COVID-19 patients who have been treated with immunosuppressants. In our case, the plausible reason for reactivation and growth of Histoplasma may be the suppression of IL6 action by Tocilizumab.
\end{abstract}

It may be prudent to screen patients for Histoplasma and other fungal infections like aspergillus before administering an immune-suppressive regimen in patients with a moderate or severe COVID19 illness. Urinary Histoplasma antigen may be used for screening in these patients. J Microbiol Infect Dis 2021; 11(3):170-173.

Keywords: COVID-19, IL6 inhibitor, Histoplasmosis

\section{INTRODUCTION}

As the pandemic of COVID-19 evolves, newer complications of the disease have come to light. There are many reports of fungal and bacterial coinfections with COVID-19 [1-3]. These coinfections maybe because of reactivation of the pre-existing or latent organisms or may be acquired afresh in the hospital. These may manifest simultaneously with the COVID-19 infection or in the recovery period. Both the disease and the treatment administered contribute to the causation. It is important to promptly recognize these infections as these coinfections lead to increased morbidity and mortality in COVID19.

\section{CASE}

A 40-years old male patient was admitted with complaints of fever with myalgia and progressive loss of weight for two months. Three months back, the patient had been treated for severe COVID-19 with bilateral pneumonia and hypoxemia at another hospital. At that time, he received Dexamethasone $4 \mathrm{mg}$ IV TDS, Tocilizumab 400mg single dose, and Remdesivir $200 \mathrm{mg}$ followed by $100 \mathrm{mg}$ for the next four days for the treatment of COVID-19. The patient became asymptomatic after three days and was discharged after seven days of asymptomatic period. At the time of discharge, RT-PCR for COVID-19 was negative, and he was afebrile. However, a day after the discharge, he developed fever again, for which he was evaluated at different facilities. Finally, after two months of continuous fever, he was admitted with us.

At the time of admission with us, the patient had tachycardia and was febrile. Systemic examination revealed enlarged spleen and 
liver. Further, round to oval, brownish scaly plaques over the trunk and the groin region were observed. A dermatology opinion was taken, and lesions were diagnosed as Tinea corporis. On investigation, he had pancytopenia with Hemoglobin of $8 \mathrm{gm} \%$, total leucocyte count of $3800 /$ cumm, and platelet count of $79000 /$ cumm. Ultrasonography (USG) of the abdomen showed hepatosplenomegaly, but no enlarged lymph nodes were reported. The patient was tested twice for COVID-19 by RTPCR, which was negative.

He was further investigated on the lines of pyrexia of unknown origin with pancytopenia. Bone marrow revealed hypocellularity with normoblastic reaction, mild increase in plasma cells, and scattered histiocytes. No atypical cells, granuloma, or parasites were seen. Serology for tropical fevers including scrub typhus, brucella, dengue, and chikungunya was negative. Malaria card test and RK39 for leishmaniasis were negative. Immunological markers including ANA, complements, RF/antiCCP, S.ACE levels were also negative. CECT chest with abdomen showed hepatosplenomegaly with small mediastinal lymphadenopathy. The patient was initially treated with empirical antibiotics for seven days, but the fever persisted. A fungal etiology was suspected in view of no response, and a serum galactomannan assay was done. Interestingly, serum galactomannan yielded positive results by both Platelia ELISA galactomannan (GMI 1.4) and IMMY sona Aspergillus galactomannan lateral flow. Both the assays showed a concordant index with $\mathrm{GMI} \geq 0.5$, yielding a positive test. There was no evident focus of invasive Aspergillosis; a PET CT was undertaken to identify the infective focus. PET-CT revealed metabolically active mediastinal, pelvic and inguinal lymph nodes, with uptake in the spleen and liver.

On day 8 of admission, the patient developed multiple tiny nodular lesions over the face (Figure 1, left panel). Because of mediastinal lymphadenopathy and new skin lesions, suspicion of Histoplasmosis was considered, and a urine sample was sent to detect Histoplasma antigen. Blood culture and punch biopsy from both the trunk and the freshly erupted face lesions were also sent for Histoplasma culture. Antigen detection for the Histoplasma galactomannan (HGM) singlemonoclonal-antibody sandwich ELISA (Immuno-Mycologics, Norman, OK) was done according to the manufacturer's instructions. According to the test validation, a cut-off of 0.5 $\mathrm{ng} / \mathrm{mL}$ was used to determine positivity. The test was reactive $(1.5 \mathrm{ng} / \mathrm{ml})$.

Meanwhile, bone marrow biopsy showed granuloma on H\&E stain (Figure 2 upper panel) and intracellular yeasts on Periodic acid Schiff's (PAS) stain (Figure 2, lower panel). PAS and Gomorri methenamine silver stain of the punch biopsy samples from the face (Figure 3), as well as the trunk, revealed small yeast cells suggestive of Histoplasma. On the $14^{\text {th }}$ day, blood culture yielded growth of Histoplasma capsulatum, which was confirmed by sequencing the ITS region. The patient was started on the definitive management for progressive disseminated Histoplasmosis with liposomal amphotericin B, following which the patient became afebrile with gradual improvement of the skin nodules (Figure 1, right panel).

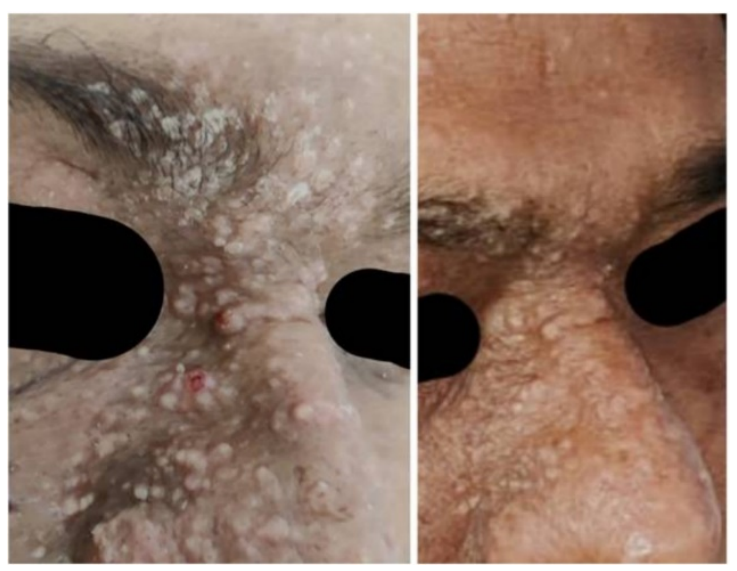

Figure 1. Skin lesions on the face before (left panel) and after treatment (right panel) with Liposomal amphotericin B.

After ten days, repeat serum galactomannan and Histoplasma antigen were done to look for the response, which showed a fall in titer to GMI-0.48 and $1 \mathrm{ng} / \mathrm{ml}$, respectively. The patient was discharged on Itraconazole after two weeks of liposomal amphotericin B and is being followed.

\section{DISCUSSION}

Histoplasmosis is a fungal disease caused by the thermally dimorphic fungus Histoplasma capsulatum, which grows in the mycelial forms in the soil [4]. Inhalation of the sporulating mycelia fragments is followed by the conversion into yeast forms and then hematogenous dissemination throughout the reticuloendothelial system [5]. There are three 
major clinical presentations of Histoplasmosis - acute pulmonary, progressive disseminated, and chronic cavitary [6]. Major risk factors for disseminated Histoplasmosis include cellular immune dysfunction, primary immune deficiency disorders, HIV, and immunosuppressive therapy [7]. The cutaneous lesions occur in $17 \%$ of the patients with disseminated Histoplasmosis and can manifest as papules, plaques, pustules, and nodules, commonly over the face, arms and legs [8].

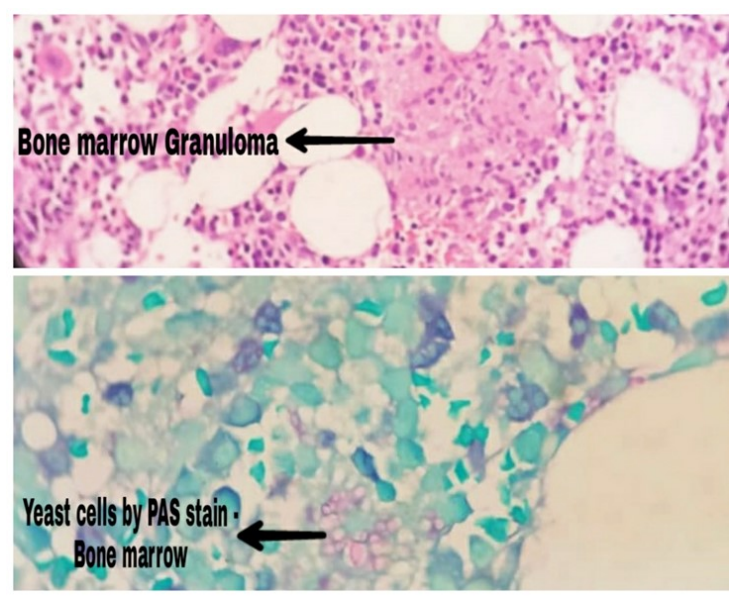

Figure 2. Bone marrow- upper panel- granuloma (H\& E stain 200X). Lower panel- intracellular yeast cells (PAS stain 200X).

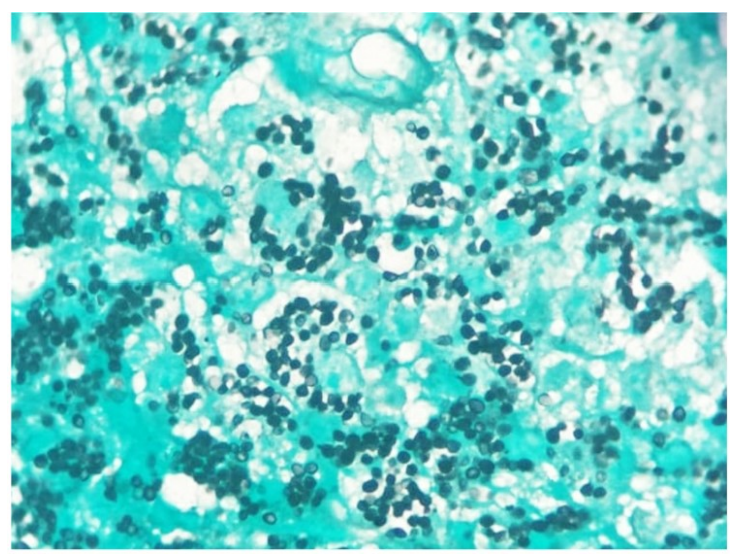

Figure 3. Skin biopsy showing intracellular yeast (Gomori methanamine silver stain 200X).

A recent review described 35 cases of COVID19 associated pulmonary aspergillosis (CAPA) [3]. COVID-19 associated ARDS, immune suppression, use of steroids, IL-6 receptor antagonists, and broad-spectrum antibiotics create an environment for developing a secondary pulmonary fungal infection. Studies are showing a substantially increased risk of candidemia and invasive Aspergillosis following Tocilizumab. In one study, among 43 patients who received Tocilizumab, three
$(6.9 \%)$ were later diagnosed with candidemia [9]. Another study on the effects of Tocilizumab in COVID-19 patients found that invasive Aspergillosis was a late complication after Tocilizumab [10].

Histoplasma coinfection with COVID-19 has been reported in two patients so far $[11,12]$. Both of them were from known endemic areas and had pre-existing HIV-AIDS. None of them had received IL6 inhibitors. IL- 6 inhibition by Tocilizumab creates a conducive environment for the reactivation and growth of Histoplasma. IL-6 plays a critical role in T cell activation and differentiation, and its absence leads to a delay in the induction of IFN-y, thus preventing the inhibition of the intracellular growth of $\mathrm{H}$. Capsulatum [13].

Keeping these in mind, we considered a fungal etiology as a cause of fever in our patient. The patient had no lung involvement but had increased uptake in the liver, spleen, and mediastinal lymph nodes on PET-CT. Our patient also had pre-existing skin lesions over the trunk, and he developed lesions over the face during the hospital stay. A possibility of reactivation of pre-existing Histoplasmosis of the skin due to immune suppression caused by covid-19 and IL6 inhibitors was considered.

The detection of the Histoplasma antigen confirmed the diagnosis of Histoplasmosis in serum/urine, visualization of yeasts cells on biopsy, isolation of Histoplasma in culture, and identification on genome sequencing. Galactomannan positivity in our case could have been because of Aspergillus Galactomannan cross-reactivity observed in patients with Histoplasmosis, and it can be used as an adjunctive test for the diagnosis of Histoplasmosis [14]. In a study by Wheat et al., it was shown that $69 \%$ of the serum sample that was positive for Histoplasma antigen were also positive for galactomannan [15].

\section{Conclusion}

To the best of our knowledge, this is the first case report of disseminated Histoplasmosis after tocilizumab therapy given for COVID-19. Thus, a possibility of disseminated Histoplasmosis in patients receiving Tocilizumab should be considered, even in non-endemic areas. Urine for Histoplasma antigen can be used for diagnosis and followup of such patients. 


\section{ACKNOWLEDGMENTS}

Declaration of Conflicting Interests: The authors declare that they have no conflict of interest.

Funding: Not applicable.

\section{REFERENCES}

1. Chen N, Zhou M, Dong X, et al. Epidemiological and clinical characteristics of 99 cases of 2019 novel coronavirus pneumonia in Wuhan, China: a descriptive study. Lancet 2020; 395:507-513

2. Garcia-Vidal C, Sanjuan G, Moreno-García E, et al. COVID-19 Researchers Group. Incidence of coinfections and superinfections in hospitalized patients with COVID-19: a retrospective cohort study. Clin Microbiol Infect. 2021; 27(1):83-88.

3. Arastehfar A, Carvalho A, van de Veerdonk FL, et al. COVID-19 Associated Pulmonary Aspergillosis (CAPA)-From Immunology to Treatment. J Fungi 2020; 6(2):91.

4. Wheat LJ. Diagnosis and management of Histoplasmosis. Eur J ClinMicrobiol Infect Dis 1989; 8:480-490.

5. Minamoto GY, Rosenberg AS. Fungal Infections in patients with acquired immunodeficiency syndrome. Med Clin North Am 1997; 81:389-409.

6. Cirillo-Hyland VA, Gross P. Disseminated histoplasmosis in a patient with acquired immunodeficiency syndrome. Cutis 1995;55(3):161164.

7. Adenis AA, Aznar C, Couppié P. Histoplasmosis in HIV-Infected Patients: A Review of New Developments and Remaining Gaps. Curr Trop Med Rep 2014;1(2):119-128.

8. Sayal S K, Prasad PS, Mehta A, Sanghi S. Disseminated Histoplasmosis: cutaneous presentation. Indian J Dermatol Venereol Leprol 2003; 69 (S1):90-1.

9. Antinori S, Bonazzetti C, Gubertini G, et al. Tocilizumab for cytokine storm syndrome in COVID19 pneumonia: an increased risk for candidemia? AutoimmunRev 2020; 19(7):102564.

10. Guaraldi G, Meschiari M, Cozzi-Lepri A, et al. Tocilizumab in patients with severe COVID-19: a retrospective cohort study. Lancet Rheumatol 2020; 2(8):474-484 .

11. Bertolini M, Mutti MF, Barletta JA, et al. COVID19 associated with AIDS-related disseminated Histoplasmosis: a case report. Int J STD AIDS 2020;31(12):1222-1224.

12. Messina FA, Marin E, Caceres $\mathrm{DH}$, et al. Coronavirus Disease 2019 (COVID-19) in a Patient with Disseminated Histoplasmosis and HIV-A Case
Report from Argentina and Literature Review. J Fungi 2020; 6(4):275.

13. Lane TE, Otero GC, Wu-Hsieh BA, Howard DH. Expression of inducible nitric oxide synthase by stimulated macrophages correlates with their antihistoplasma activity. Infect Immun 1994; 62(4):1478-9.

14. Ranque $S$, Pelletier $R$, Michel-Nguyen $A$, Dromer F. Platelia Aspergillus assay for diagnosis of disseminated histoplasmosis. Eur J ClinMicrobiol Infect Dis. 2007; 26(12):941-943.

15. Wheat LJ, Hackett E, Durkin $M$, et al. Histoplasmosis-associated cross-reactivity in the BioRadPlatelia Aspergillus enzymeimmunoassay. Clin Vaccine Immunol 2007; 14:638-640. 